This is a postprint version of the following published document:

H. Al Hajjar, C. Vázquez, D.S. Montero, P. C. Lallana, B. Fracasso "Spectral and spatial characterisation of PF-GIPOFs for the distribution of optical wireless communication cells" in Applied Optics, 542015 (5), 1138-1145. Available in http://dx.doi.org/10.1364/AO.54.001138

(C) 2015 Optical Society of America 


\title{
Spectral and spatial characterization of perfluorinated graded-index polymer optical fibers for the distribution of optical wireless communication cells
}

\author{
Hani Al Hajjar, ${ }^{1, *}$ David S. Montero, ${ }^{2}$ Pedro C. Lallana, ${ }^{2}$ \\ Carmen Vázquez, ${ }^{2}$ and Bruno Fracasso ${ }^{1}$ \\ ${ }^{1}$ Optics Department, Telecom Bretagne, Institut Telecom, CS83818, 29238 Brest Cedex 03, France \\ ${ }^{2}$ Electronics Technology Department, Universidad Carlos III de Madrid, Avenida de la Universidad, \\ 30, 28911, Leganes (Madrid), Spain \\ ${ }^{\star}$ Corresponding author: Hani.alhajjar@gmail.com
}

\begin{abstract}
In this paper, the characterization of a perfluorinated graded-index polymer optical fiber (PFGIPOF) for a high-bitrate indoor optical wireless system is reported. PF-GIPOF is used here to interconnect different optical wireless access points that distribute optical free-space high-bitrate wireless communication cells. The PF-GIPOF channel is first studied in terms of transmission attenuation and frequency response and, in a second step, the spatial power profile distribution at the fiber output is analyzed. Both character-izations are performed under varying restricted mode launch conditions, enabling us to assess the trans-mission channel performance subject to potential connectorization errors within an environment where the end users may intervene by themselves on the home network infrastructure.
\end{abstract}

\section{Introduction}

Multimode fibers (MMFs) have attracted much attention over the last years to implement short reach and medium-to-high bandwidth transmission channels. These include, for example, Gigabit Ethernet [1] and radio-over-fiber (RoF) links for in-house transmission or wireless access systems [2]. Polymer optical fibers (POFs) [3] have recently gained importance as promising transmission media for future short and medium distance home networking to replace silica-based MMF, offering such advantages as great flexibility, easy handling, and higher resilience to mechanical bending. An advanced POF generation is the perfluorinated graded-index polymer optical fiber (PF-GIPOF), based on an amorphous perfluorinated polymer showing a lower power attenuation in the infrared (IR) range [4]. This fiber combines the general aforementioned advantages of POFs with a larger capacity for data transmission [5]. Considering the PF-GIPOF as a physical transmission medium is viewed as one of the most promising solutions for the replacement of the copper cables usually deployed in customer premises networks, interconnects, and access links, whereby $40 \mathrm{Gbps}$ in a $100 \mathrm{~m}$ link at $1550 \mathrm{~nm}$ has been reported [6]. In addition to this, the increasing bandwidth demand on mobile terminals and wireless communications in various places, such as offices, homes, or airports, has led researchers and engineers to design picocell architectures, either in the form of RoF cell distribution [2] or using wireless optical communication (WOC) [7]. The latter all-optical solution exhibits some advantages as compared to 
the RoF-based option in terms of spectrum availability, data security, integration cost, limited power consumption, and immunity to electromagnetic interference, especially within indoor environments. WOC links can be implemented using either visible or IR light, but systems based on visible light communication (VLC) have drawn considerable attention over the past few years [8], due to their ability to combine lighting and data transmission. Despite its continuous performance, VLC still remains subject to bandwidth limitations, ambient light noise corruption, and poor power budget due to its relatively large diverging beam angles. As a promising alternative, especially if very high bitrates are required (several Gbps), narrow line-of-sight (NLOS) IR beams can be used, taking advantage of the performance of IR transmitter and receiver modules developed for fiber-optic systems in different wavelength bands [9-11]. The signal sources here are single-frequency lasers emitting at wavelengths ranging from 850 to $1550 \mathrm{~nm}$, with the major constraint that the power emitted in free space should respect the international standard value of eye safety conditions (class 1 laser), for example, a power limit of $10 \mathrm{dBm}$ in the $1530-1565 \mathrm{~nm}$ IR C band [12]. This value is 50 times higher than the safe power level in the visible range. In addition, the ambient light noise originating from the sun and lighting is also less harmful in the IR C band, in comparison with its level in the visible light or near-IR range (800-1100 nm). A decisive point in NLOS wireless IR systems is the difficulty to route the optical signals from the access network through free space to feed the wireless users. Among different techniques, several authors have proposed to distribute the WOC cells using a fiber infrastructure irrigating specific optical access points located in the rooms $[10,11,13]$. The role of access points is to shape and possibly steer the optical beams toward the receivers, with the main goal of maintaining a uniform SNR within each receiving cell area.

Throughout this paper, we consider an indoor fiber distribution system that transports the access network data to mobile terminals using free-space optical cells at $1550 \mathrm{~nm}$. The general architecture [11] is schematically depicted in Fig. 1. Both access traffic (fiber-to-the-home, FTTH) and locally generated home data streams are centralized using an optical control station (OCS), which can be viewed as an optical multiport switch, with possible optical amplification at that stage. At the OCS outputs, the optical signals are distributed to optical access points (OAPs) on every room, using dedicated optical fibers (possibly POF). The downstream signals are then transferred from the OAP to the mobile terminals within a room using a wireless optical link signal. Compared to existing standard optical wireless schemes using diffused links in a single room, the present fiber-distributed scheme offers NLOS transmission geometries, which enables the link power budget to be consistent with Gbps communications [14].

This argument is essential to consider a reliable and cost-effective deployment of such PF-GIPOF-based

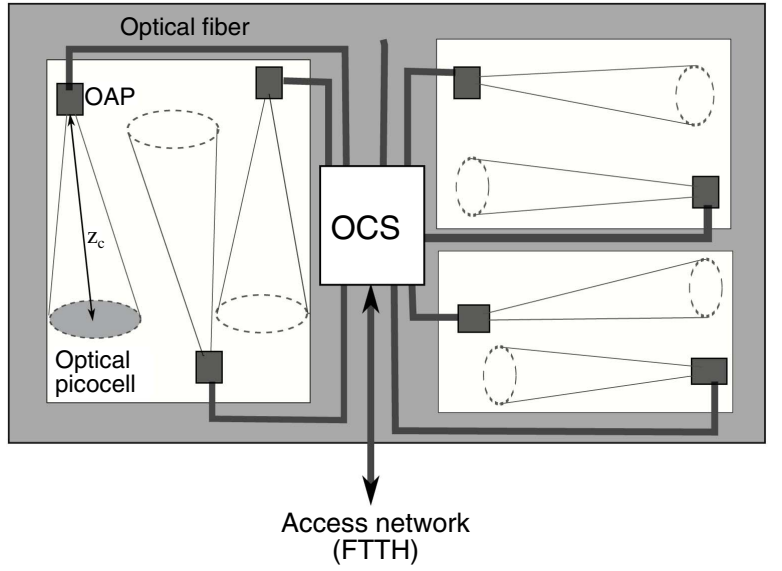

Fig. 1. Hybrid indoor optical fiber distribution system, with OCS and OAP; $z_{c}$ is the average link length between the OAP and mobile receivers, located at the optical cell.

networks and to improve the confidence of end users in the proposed architecture. Thus, in addition to meeting the above-mentioned eye-safety standards, the optical signal power delivered by the OAP in free space should be stable in time and independent from the fiber cabling and interfacing conditions. This involves a comprehensive study of the POF insertion loss and bandwidth as a function of the operating and interfacing conditions. The most sensitive parameter is probably the optical power coupling at the connection points between single-mode signals (e.g., sources) and multimode structures, known as "launch" conditions. The offset influence on bandwidth has been indirectly analyzed as a way to control launch conditions [15], but mainly at $850 \mathrm{~nm}$ wavelength and always along a single axis $[16,17]$.

In this paper, we propose an experimental characterization of the frequency and spatial properties of the optical signal emerging from a PF-GIPOF fiber subject to such varying operational conditions as the fiber length, input launch conditions, or source coherence at the two 1300 and $1550 \mathrm{~nm}$ "telecom" wavelengths. Section 2 presents a full characterization of the optical signal at the PF-GIPOF output, in terms of attenuation and frequency response for different launch conditions. The fiber far-field irradiance is assessed under varying launch conditions in Section $\underline{3}$, and a solution to produce spatially uniform optical free-space cells is provided.

\section{PF-GIPOF Attenuation Measurements}

In a highly multimode fiber like the POF, the optical signal is carried over different paths as it moves along the fiber. Each path corresponds to the propagation of an excited mode in the fiber core. The number of existing modes is variable, depending on the input launch conditions, that can be divided into two types:

- The overfilled launch condition, where the maximum number of modes is excited. This situation occurs when the launch spot size and angular distribution are larger than the fiber core, that is, for 
example, when the injection is performed using a fiber with a core diameter larger than the fiber core under test.

- The restricted mode launch (RML) condition, where a small number of modes are excited, which occurs when the launch spot size and angular distribution are smaller than the fiber core, that is, for example, when the signal is injected from a singlemode fiber (SMF). Characterizing the POF using RML is interesting to assess the fiber sensitivity to input source coupling or connector misalignment.

The fiber transmission characteristics for different launch conditions must be carefully examined to assess the fiber's performance in a real high-speed home network. It should indeed be easily installed and maintained by a nonexpert user, with, for example, a certain level of resilience performance to fiber connection errors (variation of transmission attenuation, frequency response, and so forth). This point will be studied in the next subsections.

\section{A. Fiber Loss Characterization}

PF-GIPOF presents a great advantage in terms of transmission efficiency, as compared to other polymer optical fibers, with reported linear attenuation coefficients (per $100 \mathrm{~m}$ ) of $1,1.5$, and $15 \mathrm{~dB}$, measured respectively at 1000,1300 , and $1550 \mathrm{~nm}$ [18]. The stability of the transmission attenuation value of a PF-GIPOF span can be evaluated through the experimental measurement of the fiber loss under different RML launch conditions, where an offset from the fiber axis is supposed to simulate a connection misalignment. This experiment consists in injecting a monochromatic and single-mode optical signal at different points of the larger input PF-GIPOF core and measuring the variations of the signal power at the fiber output. Considering power values expressed in logarithmic units $(\mathrm{dBm})$, the difference between the input and output fiber power values will be referred to as insertion loss (IL). Although this is not a general rule, minimal IL values are expected for RML launch conditions at the center of the fiber core. Then, under current shifted RML conditions, the excess loss (EL) is defined as the difference between the IL with center RML conditions and the IL with the shifted RML conditions. Finally, the Allowable Offset Launch Tolerance (AOLT) will be chosen as the offset launch distance limit that generates EL values lower than $1 \mathrm{~dB}$, which is considered as a suitable metric for the fiber connectorization sensitivity. The fiber EL for varying RML conditions and the resulting allowable offset launch tolerance are evaluated here for 50 and $62.5 \mu \mathrm{m}$ core diameter fibers. Fiber lengths of 50 and $100 \mathrm{~m}$ and two operating 1300 and $1550 \mathrm{~nm}$ wavelengths are considered to determine the range of suitable performance for the proposed architecture. The setup used is shown in Fig. 2. The output of the optical laser source is connected to a SMF through a $95: 5$ coupler. The $5 \%$ output power port is used to monitor the optical power source stability, and the $95 \%$ output power signal is injected in the

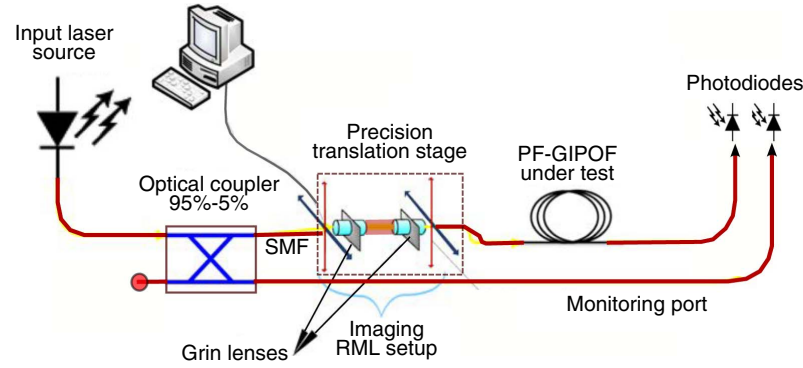

Fig. 2. Experimental setup to measure the PF-GIPOF IL under varying offset launch conditions.

PF-GIPOF through a dedicated imaging setup involving two positive gradient index (GRIN) lenses in a $4 f$ setup. A three-axis precision translation stage is used to scan the position of the SMF fiber, with a step resolution of $0.2 \mu \mathrm{m}$ and bidirectional repeatability of $3 \mu \mathrm{m}$. At the receiving stage, the optical power emerging from the PF-GIPOF is measured by an InGaAs photodetector, supporting the large core size of a multimode optical fiber. The translation stage is equipped with three linear actuators (Newport CMA-25CCCL) controlled by a computer program under LabVIEW. The tests are performed using a $1300 \mathrm{~nm}$ Fabry-Perot laser source and a $1550 \mathrm{~nm}$ DFB laser. Two orthogonal radial offsets are scanned along the entire PFGIPOF end face under test, with $5 \mu \mathrm{m}$ increments, and the output power versus the input offset launch is measured after 50 and $100 \mathrm{~m}$ propagation lengths, respectively. These distances are suitable for most commonly deployed indoor optical cabling solutions. The PF-GIPOF samples used were manufactured by Chromis Fiberoptics, Inc. [19]. Initially, the input SMF is positioned perpendicular to the PF-GIPOF input end face and at the center of the fiber core. It is supposed that the maximum transmitted power occurs at this particular location, with a negligible error of $3 \mu \mathrm{m}$, corresponding to the repeatability step of the precision translation stage.

Figure 3 shows the measured EL curves along $100 \mathrm{~m}$ of $\overline{\mathrm{P}} \mathrm{F}$-GIPOF at $1550 \mathrm{~nm}$ wavelength for 50 and $62.5 \mu \mathrm{m}$ core diameters and two $X$ and $Y$ orthogonal axes [20]. It shows that $1 \mathrm{~dB}$ EL limits a $\pm 10 \mu \mathrm{m}$ zone around the fiber axis. Table 1 summarizes the set of parameters measured at both 1300 and $1550 \mathrm{~nm}$ for the PF-GIPOF samples under test. The ratio between the AOLT and the fiber core diameter $a$ is considered as a quantifying parameter of how efficiently light can be injected into the fiber under test, with regards to its diameter. We also estimate the slope $\sigma$ of the variation of the EL at specific points where the EL value is $3 \mathrm{~dB}$.

As expected, we can notice that the IL values are higher at $1550 \mathrm{~nm}$ than at $1300 \mathrm{~nm}$. It also appears that longer PF-GIPOF lengths become slightly less tolerant to offset launching conditions. This point can be easily noticed on the AOLT/ $a$ ratio, for which the average measured values ( $x$ axis) between 50 and $100 \mathrm{~m}$ fiber samples vary from $58 \%$ to $46 \%$ at $1300 \mathrm{~nm}$, respectively, and from $67 \%$ to $58 \%$ at 


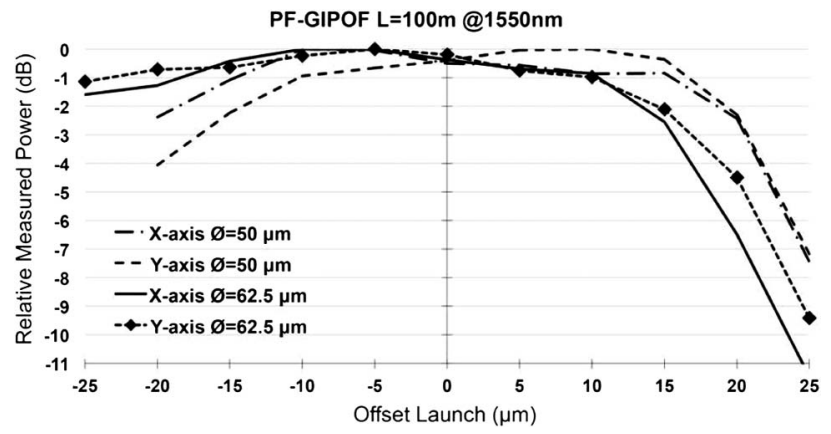

Fig. 3. Relative measured power at the PF-GIPOF output for different offset launch conditions comparison between two core diameters for a $100 \mathrm{~m}$ length and $1550 \mathrm{~nm}$ of wavelength along two orthogonal axes.

$1550 \mathrm{~nm}$, respectively. Minor variations noticed for the $y$ axis are due to fiber imperfections during manufacturing. In addition, EL values at $1300 \mathrm{~nm}$ are higher as compared to the values obtained at $1550 \mathrm{~nm}$. This can be noticed on the AOLT/ $a$ values measured for $100 \mathrm{~m}$ fiber length, varying from $50 \%$ (50 $\mu \mathrm{m}$ core) to $42 \%(62.5 \mu \mathrm{m})$ at $1300 \mathrm{~nm}$, and from $58 \%(50 \mu \mathrm{m}$ core $)$ to $59 \%(62.5 \mu \mathrm{m})$ at $1550 \mathrm{~nm}$. This can be explained by differential mode attenuation effects, which are supposed to be stronger at $1300 \mathrm{~nm}$ as compared to the situation at $1550 \mathrm{~nm}$. Concerning the slope metric, the general trend follows what could be expected, that is, higher slopes are measured at $1300 \mathrm{~nm}$ and for longer fiber lengths. These first results are complemented by more detailed attenuation measurements, for input RML conditions sweeping over the whole input fiber core area. For this purpose, an automated full 2D scan of the fiber core is performed to measure the EL fluctuations through $50 \mathrm{~m}$ of PF-GIPOF, covering an offset range of $\pm 25 \mu \mathrm{m}$ with $5 \mu \mathrm{m}$ steps in both dimensions. Figures $\underline{4}$ and $\underline{5}$ show the resulting excess power loss maps measure $\bar{d}$ for 50 and $62.5 \mu \mathrm{m}$ core diameters, at

Table 1. Experimental Metrics Measured to Evaluate the Offset Launch Sensitivity, at Two Wavelengths and for Different Fiber Lengths and Core Diameter

\begin{tabular}{|c|c|c|}
\hline Operating Wavelength & \multicolumn{2}{|c|}{$1300 \mathrm{~nm}$} \\
\hline Fiber length & $50 \mathrm{~m}$ & $100 \mathrm{~m}$ \\
\hline Core diameter $a(\mu \mathrm{m})$ & $50 / 62.5$ & $50 / 62.5$ \\
\hline Center attenuation $(\mathrm{dB})$ & $1.9 / 2.4$ & $3.7 / 5.6$ \\
\hline dB X-AOLT $(\mu \mathrm{m})$ & $\pm 15 / \pm 17.5$ & $\pm 12.5 / \pm 13$ \\
\hline$(\mathrm{AOLT} / a)$ & $(60 \% / 56 \%)$ & $(50 \% / 42 \%)$ \\
\hline dB Y-AOLT $(\mu \mathrm{m})$ & $\pm 13 / \pm 16$ & $\pm 12 / \pm 15.5$ \\
\hline$(\mathrm{AOLT} / a)$ & $(52 \% / 51 \%)$ & $(48 \% / 49 \%)$ \\
\hline Slope $\sigma$ at $3 \mathrm{~dB}$ fall $(\mathrm{dB} / \mu \mathrm{m})$ & $-1.22 /-0.40$ & $-1 /-0.88$ \\
\hline Operating Wavelength & \multicolumn{2}{|c|}{$1550 \mathrm{~nm}$} \\
\hline Fiber length & $50 \mathrm{~m}$ & $100 \mathrm{~m}$ \\
\hline Core diameter $a(\mu \mathrm{m})$ & $50 / 62.5$ & $50 / 62.5$ \\
\hline Center attenuation $(\mathrm{dB})$ & $14.1 / 14.7$ & $27.9 / 28.0$ \\
\hline dB X-AOLT $(\mu \mathrm{m})$ & $\pm 17.5 / \pm 20$ & $\pm 14.5 / \pm 18.5$ \\
\hline$(\mathrm{AOLT} / a)$ & $(70 \% / 64 \%)$ & $(58 \% / 59 \%)$ \\
\hline dB Y-AOLT $(1 \mathrm{~dB})(\mu \mathrm{m})$ & $\pm 13.5 / \pm 18$ & $\pm 14 / \pm 17.5$ \\
\hline$(\mathrm{AOLT} / a)$ & $(54 \% / 58 \%)$ & $(56 \% / 56 \%)$ \\
\hline Slope $\sigma$ at $3 \mathrm{~dB}$ fall $(\mathrm{dB} / \mu \mathrm{m})$ & $-0.83 /-0.50$ & $-0.98 /-0.79$ \\
\hline
\end{tabular}
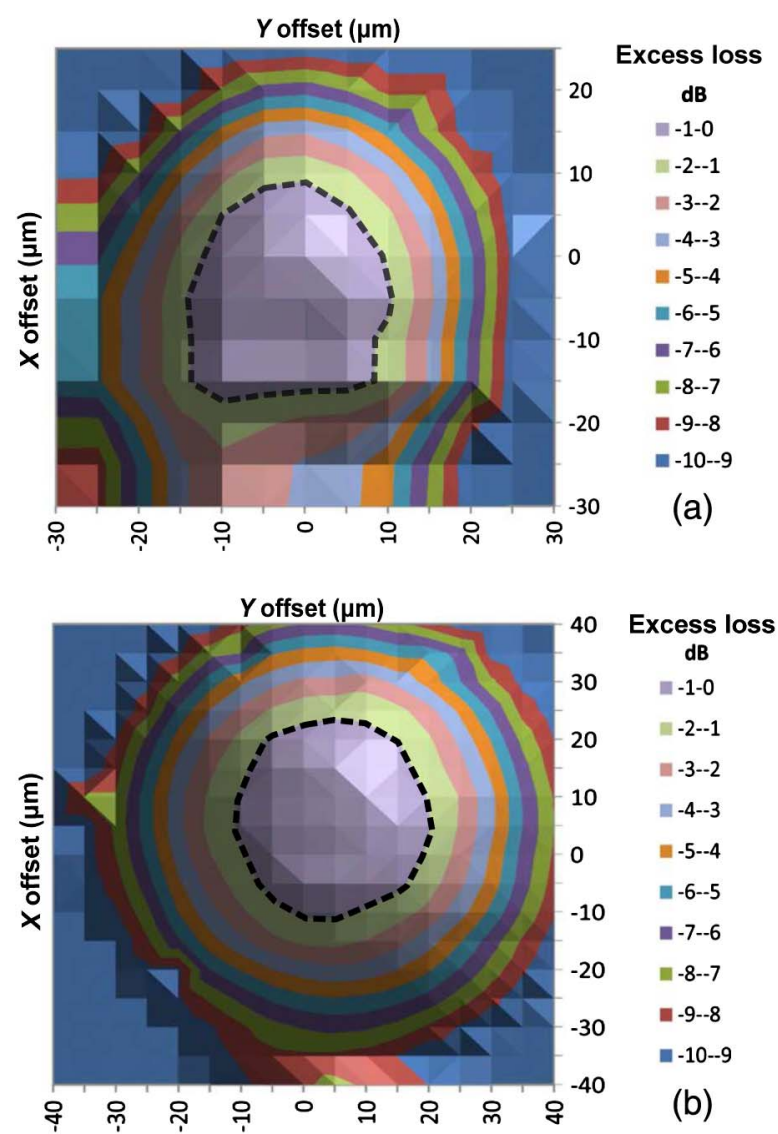

Fig. 4. Measured power EL for a RML sampling grid with a $5 \mu \mathrm{m}$ step at $1300 \mathrm{~nm}$ for (a) $50 \mu \mathrm{m}$ and (b) $62.5 \mu \mathrm{m}$. PF-GIPOF length is $50 \mathrm{~m}$ and the dashed line indicates the AOLT limit.

1300 and $1550 \mathrm{~nm}$, respectively. In both cases, the $1 \mathrm{~dB}$ EL limits a quasi-circular zone with a radius exceeding $10 \mu \mathrm{m}$ around the fiber center location, which gives an estimate of the fiber connection misalignment tolerance that could be allowed in a real system environment. This value is consistent with the positioning repeatability of standard POF connectors like, for example, the SC standard.

\section{B. Frequency Response Measurement}

The ability of the medium to transmit very high bitrates is now assessed. To this aim, the POF frequency response is measured using a light wave component analyzer (Agilent 8703B), as shown in Fig. $\underline{6}$. For the two operational wavelengths, the lasers are externally amplitude modulated using a $16 \mathrm{GHz}$ bandwidth electro-optic (EO) Mach-Zehnder modulator (JDSU AM-130 at $1300 \mathrm{~nm}$ and JDSU AM-155 at $1550 \mathrm{~nm})$. At the receiver stage, a high-speed PIN photodiode (DSC30 S, from Discovery Semiconductors) is used to detect the variations of the optical signal average power. This device is specifically designed for high-speed multimode fiber transmission, with $24 \mathrm{GHz}$ bandwidth at $-3 \mathrm{~dB}$ loss. Before introducing the PF-GIPOF span, the experimental link is calibrated "back-to-back" with regard to the electrical responses of both the EO intensity modulator and the photodetector. The fiber frequency response is 

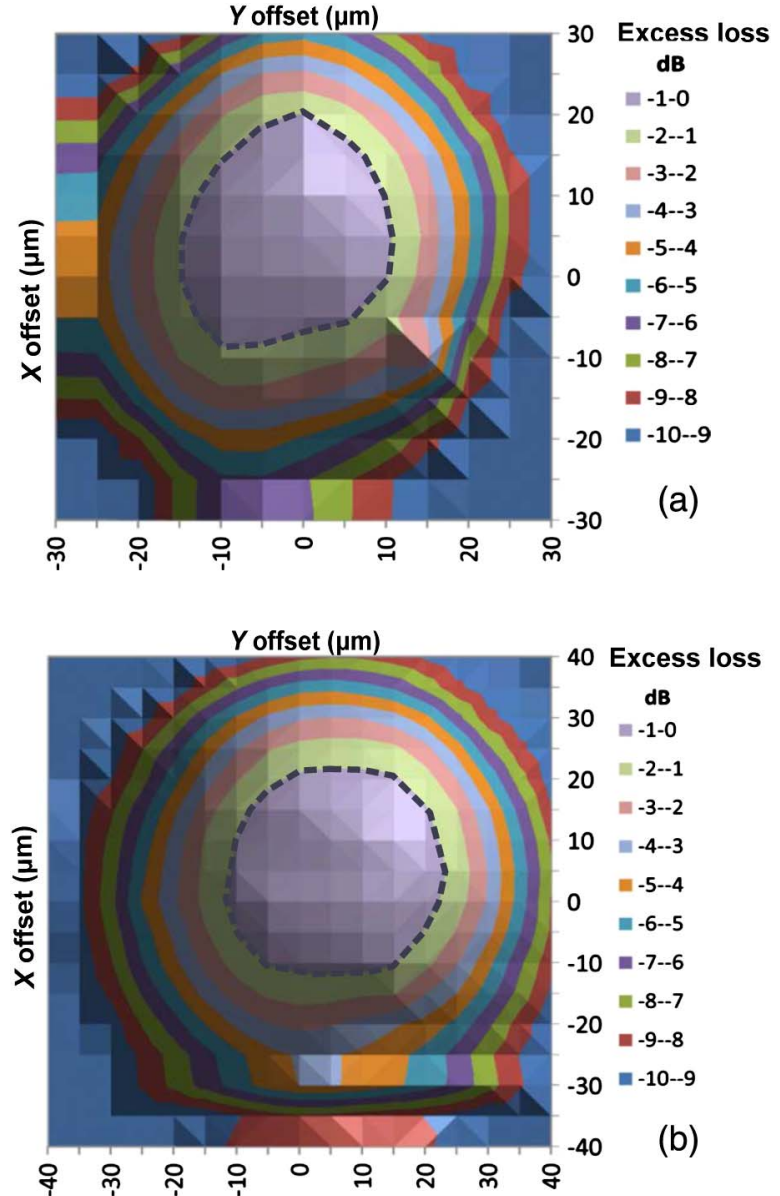

Fig. 5. Measured power EL for a RML sampling grid with a $5 \mu \mathrm{m}$ step at $1550 \mathrm{~nm}$ for (a) $50 \mu \mathrm{m}$ and (b) $62.5 \mu \mathrm{m}$. PF-GIPOF length is $50 \mathrm{~m}$ and the dashed line indicates the AOLT limit.

evaluated along two orthogonal radial axes for a $\pm 25 \mu \mathrm{m}$ offset range with $5 \mu \mathrm{m}$ steps. The bandwidth measurement results at the output of $50 \mathrm{~m}$ long and 50 and $62.5 \mu \mathrm{m}$ core diameter fiber are given in Fig. 7 , for the two 1300 and $1550 \mathrm{~nm}$ wavelengths. The experimental curves show a $-3 \mathrm{~dB}$ bandwidth exceeding $2.5 \mathrm{GHz}$ for an offset margin of $\pm 15 \mu \mathrm{m}$.

Using such a standard modulation scheme as non-return-to-zero on-off keying (NRZ-OOK), this $2.5 \mathrm{GHz}$ channel bandwidth would ensure the transmission of multi-Gbps bitrates over at least $50 \mathrm{~m}$ links, which is a suitable distance for in-house network deployments. This result confirms the option

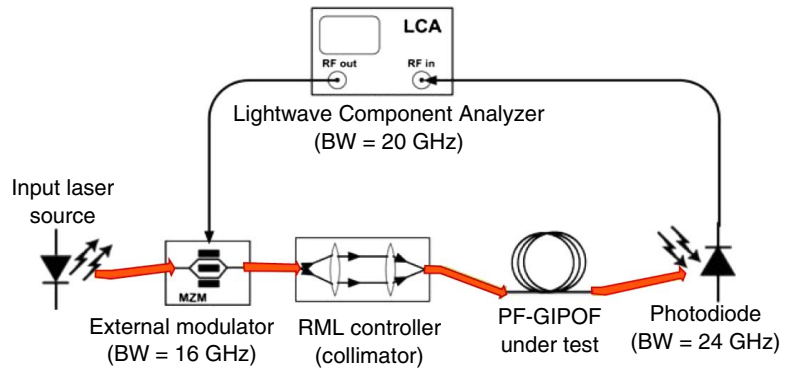

Fig. 6. Experimental setup to measure the PF-GIPOF frequency response under varying offset launch conditions.

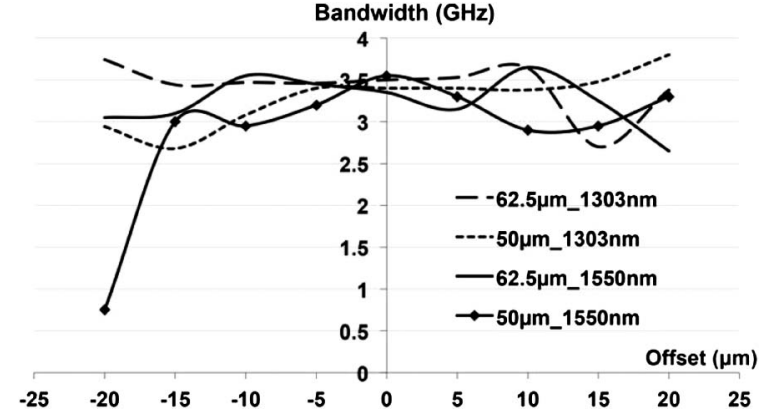

Fig. 7. Variation of the $-3 \mathrm{~dB}$ bandwidth over $50 \mathrm{~m}$ of PF-GIPOF as a function of the offset launch for two different core diameters and two operating wavelengths.

of using the PF-GIPOF in a high-bitrate in-house transmission system, whereby the installation by the end user increases the probability of connection misalignment.

\section{Far-Field Characterization and Beam Shaping}

In the distribution architecture shown in Fig. 1, the free-space link geometry between the OAP and the receiver is supposed to be NLOS to avoid multipath distortion and minimize power loss due to free-space propagation. The optical signal emerging from the fiber must therefore be adapted in divergence, uniformity, and position to cover a picocell area, via collimation and possibly beam deflection. The typical NLOS indoor link length is here $z_{c}=2.5 \mathrm{~m}$ (Fig. 1), yielding an optical cell diameter of $\approx 15 \mathrm{~cm}$ at the receiver (see [14]). An important criterion here is the power uniformity at the receiving cell, which must be as high as possible to guarantee a constant SNR, and hence, a constant bit-error rate. However, highly multimode fibers as POFs in association with narrow linewidth lasers are known to produce speckle-like output patterns, due to random interferences among the many guided modes propagating along different paths and with different group velocities. The evolution of the speckle contrast along the fiber length is strongly related to the mode coupling between the propagation modes, which is strong in the PFGIPOF. At the fiber output, the highest-order mode has an emerging critical angle $\theta_{m}$, with $\sin \theta_{m}=\mathrm{NA}$ the fiber numerical aperture. Therefore, given the basic law of coherently interfering waves at angle $\theta_{m}$, the finest speckle size $\delta_{s}$ at the fiber output is approximately given by half the fringe spacing, that is, $\delta_{s}=\lambda / 2 \mathrm{NA}$. All intermediate modes emerge in a $2 \mathrm{D}$ angular distribution, and the corresponding beams interfere at angles between 0 and $\theta_{m}$ (yielding lower spatial frequencies), but the finest structure of the fringe pattern can still be estimated by $\delta_{s}$. On top of this static description, it has been shown that the phenomenon is not stable in time. Such speckle fluctuations (modal noise) vary with respect to fiber temperature, bending, and stress as well as the input launch conditions [21], depending on both the spectral characteristics of the laser source and the quality of the connection between the source and the 
fiber. Constructive and destructive mode coupling is particularly noticeable for the short fiber lengths used here, as the modal equilibrium may not have been reached during propagation.

When radiating from the fiber into the surrounding air medium, the light beam diffracts and the resulting electromagnetic field evolves from a nearfield distribution, close to the fiber end face, into a far-field distribution farther away. An estimation of the far-field limit is the Rayleigh length, which, in the present case, can be estimated by $z_{R}=\pi \omega_{0}^{2} / \lambda$, where $\omega_{0}$ is the radius of the beam emerging from the fiber. For the present PF-GIPOFs, $\omega_{0} \approx 30 \mu \mathrm{m}$ and the Rayleigh length is well under the $1 \mathrm{~mm}$ value. Beyond the Rayleigh length, the beam will expand at a constant divergence (numerical aperture), and the beam profile at the receiving cell distance $z_{c}$ will be estimated using a scaling factor imposed by the far-field diffraction process. Thus, the uniformity of the cell power distribution can be estimated by measuring the far-field distribution emerging from the fiber output. The far-field irradiance pattern acquisition can be obtained either by a scanning angular $1 \mathrm{D}$ method or using $2 \mathrm{D}$ image acquisition with a $2 \mathrm{D}$ sensor. We shall use the second method, as it is much faster than the scanning one and avoids the vertical prealigning of the detecting system with the fiber axis to determine the far-field pattern (FFP) diameter.

The acquisition setup geometry is shown in Fig. $\underline{8}$, with an acquisition distance $z_{s}$ of the diffracted beam with divergence angle $\alpha=\sin ^{-1}(\mathrm{NA})$. The $z_{s}$ distance is set in the far-field range $\left(z_{s} \gg z_{R}\right)$ so that the beam covers a large part of the sensor diameter $\phi_{s}$, that is, $z_{s} \approx \phi_{s} / 2 \alpha$, using a small angle approximation. The offset launching conditions are controlled here with the same coupling system as that used in Section 2. A dedicated positioning minibench is used to hold the $2 \mathrm{D}$ sensor, the $z$ axis translation stage, the fiber holder attached to an $x y$ translation stage, and a foundation plate to support the different elements.

FFP is measured using an InGaAs CMOS camera (Hamamatsu C10633-13) with high sensitivity in the 900-1700 nm IR region. The sensor is a $320 \times 256$ and $30 \mu \mathrm{m}$ pitch pixel array, and the 14-bit image acquisition plane is connected to a computer through a USB port. Far-field measurement profiles are performed for a $50 \mu \mathrm{m}$ core and $50 \mathrm{~m}$ long PF-GIPOF. Three different lasers sources are used at 1300, 1535, and $1550 \mathrm{~nm}$ wavelengths, using offset launch variations ranging from 0 to $10 \mu \mathrm{m}$, with respect to the fiber axis. The measured $-3 \mathrm{~dB}$ spectral widths of the

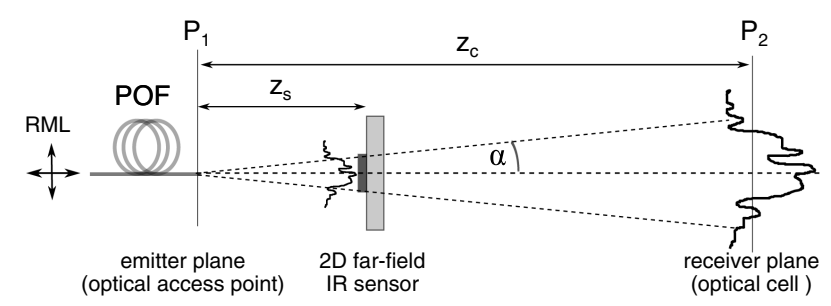

Fig. 8. FFP acquisition geometry.

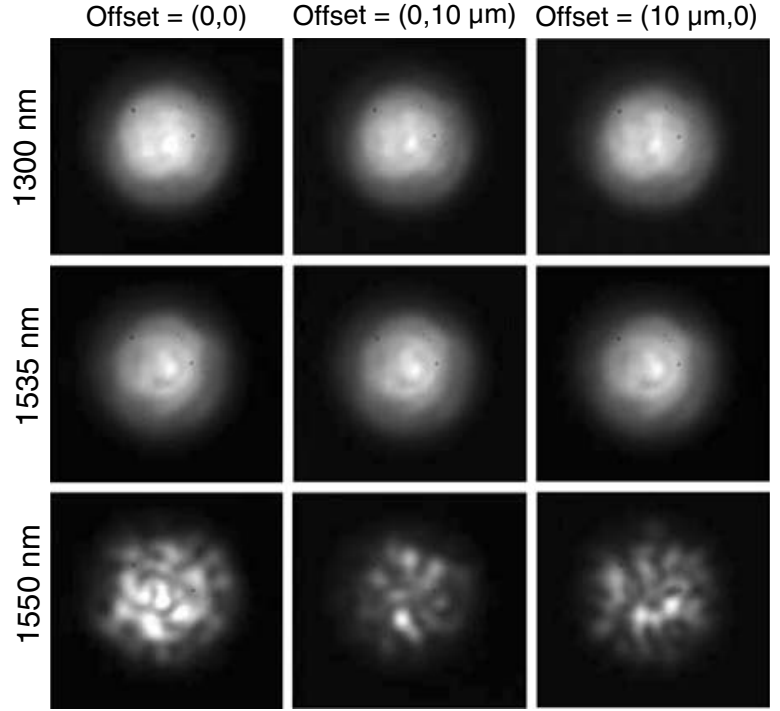

Fig. 9. Experimental irradiance distributions at the output of a $50 \mathrm{~m}$ PF-GIPOF with $50 \mu \mathrm{m}$ core diameter, using three input IR laser sources and under three different input RML conditions.

1300, 1535, and $1550 \mathrm{~nm}$ lasers are 5, 2, and $1 \mathrm{pm}$, respectively. The captured far-field irradiance maps are shown in Fig. 9 . We observe the impact of the input source spectral width, whereby a larger coherence time generates a more contrasted speckle pattern. However, the dependence on the offset launching conditions is weak, in both directions.

The propagation of the beams shown in Fig. 9 over the free-space link distance $\left(z_{c} \approx 2.5 \mathrm{~m}\right)$ with no correction at the emission would induce both too large a cell diameter $(\approx 1 \mathrm{~m})$ and a Gaussian envelope with unacceptable time-varying "speckle" fluctuations in beam power. It is possible to solve these two problems simultaneously using a custom spatial beamshaping optical element. For both flexibility and fabrication constraints, this function is advantageously performed using diffractive optical elements (DOEs), in the form of $2 \mathrm{D}$ phase gratings, working through the juxtaposition of diffraction orders. The design, fabrication, and test of this solution are out of the scope of this paper and this work has been submitted for publication elsewhere [22]. Briefly speaking, the operation is depicted in Fig. 10(a). The far-field DOE design is based on the Fourier transform between the two conjugate planes $\left(P_{1}\right.$ at the OAP and $P_{2}$ at the wireless receiving cell). In that configuration, a compact Fourier reconstruction setup based on a positive lens (with $f$ focal length) focuses the beam emerging from the fiber at $\mathrm{P}_{1}$ (distance $f+d$ ) in the $\mathrm{P}_{2}$ cell plane, located at a distance $z_{c}$ away from $P_{1}$. The DOE array illuminator is designed so as to generate a set of regular and uniform diffraction orders arranged in a disk configuration. Placing the component after the lens will spatially replicate the Fourier distribution of the fiber far field along the sampling grid formed by the diffraction orders of the DOE.

We computed a binary-phase DOE generating $N=$ 31 diffraction orders along the diameter of the 
(a)

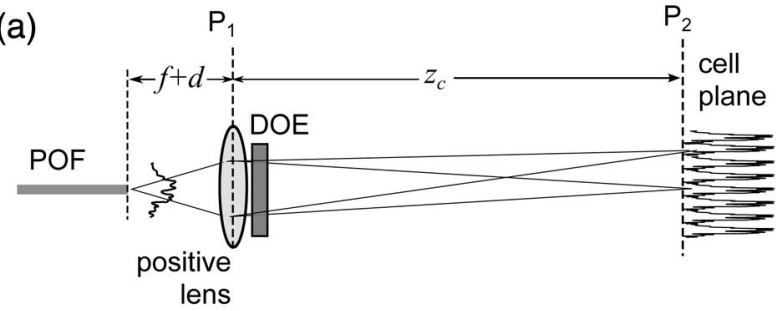

(b)

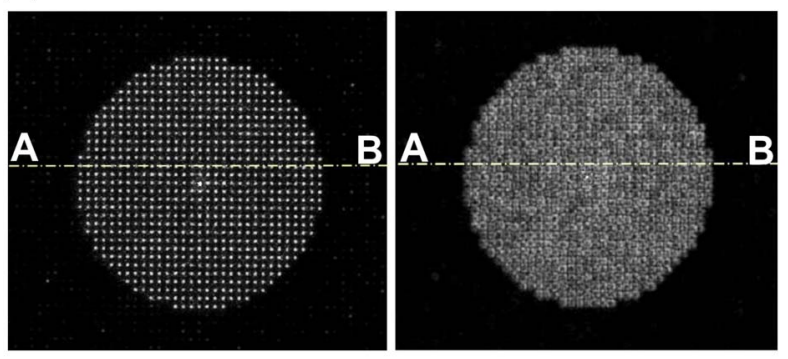

(c)

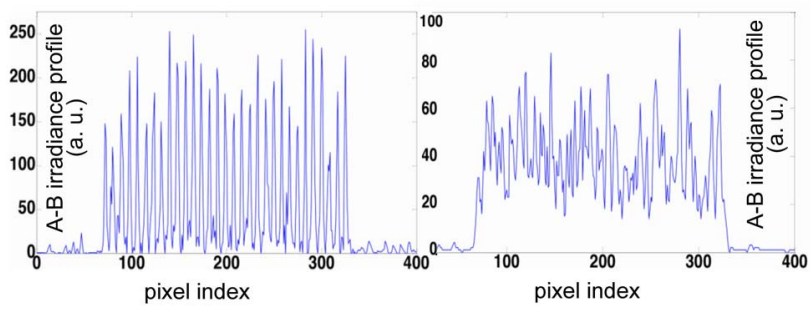

Fig. 10. (a) Beam-shaping setup to be used at the optical access point; (b) reconstructed optical cells using a beam-shaping EOD generating a disk-shaped diffraction pattern containing 31 orders in its diameter in the focused configuration (left) and homogenized/ blurred configuration (right); and (c) A-B line profiles proportional to the captured beam irradiance are given as a function of the pixel index.

circular cell. We fabricated the component in a photoresist layer on a glass substrate by massively parallel direct write, and this master structure was copied into a commercial sol-gel ormocomp layer (micro resist technology $\mathrm{GmbH}$ ) by nanoimprint, a technique that can be used for mass production [23]. Figure 10 (b) (left) shows the focused optical reconstruction generated in the $P_{1}$ Fourier plane under illumination at $\lambda_{0}=1550 \mathrm{~nm}$ using the laser source that gives the irradiance distributions in Fig. 9. The IR beam irradiance is captured using the above-mentioned InGaAs CMOS camera. The overall number of diffraction orders located in the circular cell can be estimated by $\pi N^{2} / 4 \approx 760$. The $\pi$ phase shift of the binary DOE is optimized at $\lambda_{0}$ so that the zero-order diffracted light is very weak, increasing the average irradiance value over the cell. This point is also crucial for an eye-safety configuration. The DOE efficiency $\eta$ is defined as the ratio of the overall power diffracted within the circular area to the illuminating optical power. In the present case, we have measured $\eta=71 \%$, corresponding to a moderate $1.5 \mathrm{~dB}$ power loss, for which half could be saved using antireflection coatings at the DOE faces. The optical reconstruction can be homogenized by shifting the $d$ defocusing parameter, which produces a blurring effect on the focused spots. Figure 10(b) (right) presents a view of the corresponding light distribution in the reconstruction plane with $d=20 \mu \mathrm{m}$. The beam uniformity has been clearly improved with respect to the focused configuration $(d=0)$, which is confirmed by the gray level line profiles displayed as a function of the pixel index and proportional to the beam irradiance captured [Fig. 10(c)]. Such a homogeneous distribution is well adapted to produce a constant SNR when a high-speed photoreceiver including an optical concentrator is used at the cell plane [24].

\section{Conclusion}

We have proposed a full characterization of PFGIPOF fibers in the context of in-house high-speed optical wireless link distribution. For that purpose, the fiber EL, $3 \mathrm{~dB}$ bandwidth, and far-field homogeneity have been measured experimentally for different fiber core diameters $(50$ and $62.5 \mu \mathrm{m})$, fiber lengths (50 and $100 \mathrm{~m}$ ), input laser source wavelength (1300 and $1550 \mathrm{~nm}$ ), and RML offset-launch conditions. We have shown that in most configurations, including a full 2D RML sweep, the $1 \mathrm{~dB}$ EL map limits a quasi-circular zone with a radius exceeding $10 \mu \mathrm{m}$ around the fiber center location, which is consistent with the positioning repeatability of standard POF connectors. It is, however, worth noting that larger power attenuation at $1550 \mathrm{~nm}$ will require higher launched power at the fiber input, that is, $17 \mathrm{dBm}$ to produce the eye-safety $10 \mathrm{dBm}$ limit at the output of a $50 \mathrm{~m}$ fiber link. Then, the fiber bandwidth characterization has shown that $2.5 \mathrm{GHz}$ are available over $50 \mathrm{~m}$ links, whatever the wavelength and input RML conditions under the $1 \mathrm{~dB}$ EL limit. This clearly confirms the possibility of using the PF-GIPOF medium to transport multi-Gbps data streams. Finally, we have provided qualitative evidence that the characteristics of the speckle pattern generated at the fiber output and propagating to the far field are more closely related to the input source coherence than to the input RML conditions. To avoid such beam inhomogeneities generating a fluctuating signal irradiance at the receiving cell, we have presented a custom beam-shaping method at the fiber output, based on a circular array generator binaryphase DOE with $71 \%$ diffraction efficiency. Irradiance profile acquisitions using a defocused configuration show that the beam spatial homogeneity is greatly improved. Work is in progress to characterize the quality of the resulting detected electrical signal when a high-speed photodetector coupled to an optical concentrator is used at the receiver. First results show that the SNR is almost constant and consistent with multi-Gbps communications, using standard modulation schemes.

This work has been partially supported by the Collège Doctoral International/Université Européenne de Bretagne (CDI/UEB) and "Conseil Régional de Bretagne" and by the Spanish Ministry of Economía y Competitividad under the grant TEC2012-37983C03-02. 


\section{References}

1. A. Flatman, "In-premises optical fibre installed base analysis to 2007," presented at the IEEE 802.3 10GbE over FDDI Grade Fibre Study Group, Orlando, Florida, 2004.

2. M. C. Parker, S. D. Walker, R. Llorente, M. Morant, M. Beltran, I. Mollers, D. Jager, C. Vázquez, D. S. Montero, I. Libren, S. Mikroulis, S. Karabetsos, and A. Bogris, "Radio-over-fibre technologies arising from the building the future optical network in Europe (BONE) project," IET Optoelectron. 4, 247-259 (2010).

3. J. Meier, W. Lieber, W. Heinlein, W. Groh, P. Herbrechtsmeier, and J. Theis, "Time-domain bandwidth measurements of stepindex plastic optical fibres," Electron. Lett. 23, 1208-1209 (1987).

4. T. Ishigure, A. Horibe, E. Nihei, and Y. Koike, "Highbandwidth, high-numerical aperture graded index polymer optical fiber," IEEE J. Lightwave Technol. 13, 1686-1691 (1995).

5. T. Ishigure, Y. Aruga, and Y. Koike, "High-bandwidth PVDFclad GI POF with ultra-low bending loss," IEEE J. Lightwave Technol. 25, 335-345 (2007).

6. Y. Koike and K. Koike, "Progress in low-loss and highbandwidth plastic optical fibers," J. Polym. Sci. B 49, 2-17 (2011).

7. K.-D. Langer, J. Grubor, O. Bouchet, M. El Tabach, J. W. Walewski, S. Randel, M. Franke, S. Nerreter, D. C. O'Brien, G. E. Faulkner, I. Neokosmidis, G. Ntogari, and M. Wolf, "Optical wireless communications for broadband access in home area networks," in Proc. 10th International Conference on Transparent Optical Networks (ICTON), Athens, Greece, 22-26 June 2008, pp. 149-154.

8. H. Elgala, R. Mesleh, and H. Haas, "Indoor optical wireless communication: potential and state-of-the-art," IEEE Commun. Mag. 49(9), 56-62 (2011).

9. H. Le Minh, C. C. O’Brien, G. Faulkner, O. Bouchet, M. Wolf, L. Grobe, and J. Li, "A 1.25 Gbps indoor cellular optical wireless communications demonstrator," IEEE Photon. Technol. Lett. 22, 1598-1600 (2010)

10. K. Wang, A. Nirmalathas, C. Lim, and E. Skafidas, "Highspeed optical wireless communication system for indoor applications," IEEE Photon. Technol. Lett. 23, 519-521 (2011).

11. H. A. Hajjar, B. Fracasso, and D. Leroux, "Fiber-distributed indoor high bitrate optical wireless system," presented at the 14th International Symposium on Wireless Personal Multimedia Communications (WPMC), Brest, France, October 2011.
12. European Standard EN 60825-1:2007, 2nd ed., "Safety of laser products-part 1: equipment classification and requirements," 2007.

13. C. W. Oh, F. M. Huijskens, Z. Cao, E. Tangdiongga, and A. M. J. Koonen, "Toward multi-Gbps indoor optical wireless multicasting system employing passive diffractive optics," Opt. Lett. 39, 2622-2625 (2014).

14. H. A. Hajjar, B. Fracasso, and D. Leroux, "Indoor distributed optical wireless Gbps link dimensioning," presented at the Optical Fiber Communications Conference (OFC), Anaheim, California, March 2013.

15. L. Raddatz, I. H. White, D. G. Cunningham, and M. C. Nowell, "An experimental and theoretical study of the offset launch technique for the enhancement of the bandwidth of multimode fiber links," IEEE J. Lightwave Technol. 16, 324-331 (1998).

16. C. Lethien, C. Loyez, and J. P. Vilcot, "Differential mode delay measurements of fluorinated graded index polymer optical fiber," IEEE Photon. Technol. Lett. 20, 1584-1586 (2008).

17. P. J. Decker, A. Polley, J. H. Kim, and S. E. Ralph, "Statistical study of graded-index perfluorinated plastic optical fiber," J. Lightwave Technol. 29, 305-315 (2011).

18. Y. Koike and T. Ishigure, "High-bandwidth plastic optical fiber for fiber to the display," J. Lightwave Technol. 24, 4541-4553 (2006).

19. http://www.chromisfiber.com/datacenter.htm.

20. H. A. Hajjar, D. S. Montero, P. Lallana, B. Fracasso, and C. Vázquez, "Offset-launch characterization of transmission loss in PF-GIPOF for wireless home networks," presented at the International Conference on Plastic Optical Fibers 2011, Bilbao, Spain, September 2011.

21. M. Imai, "Statistical properties of optical fiber speckles," Bull. Fac. Eng. 130, 89-104 (1986).

22. H. A. Hajjar, B. Fracasso, and K. Heggarty, "Uniform freespace cell shaping using diffractive-type beam diffusers for optical wireless links," Opt. Lett. 39, 6871-6874 (2014).

23. A. Chan Yong, K. Heggarty, C. Carre, and D. Battarel, "Rapid prototyping of diffractive optical elements in microstructured sol-gel hybrid material," presented at the 2nd EOS Conference on Manufacturing of Optical Components, Munich, 23-25 May 2011.

24. H. A. Hajjar, B. Fracasso, and F. Lamarque, "Mini optical concentrator design for indoor high bit rate optical wireless communications," presented at the 2nd International Workshop on Optical Wireless Communications, IWOW13, Newcastle, UK, 21 October 2013. 